\title{
RENDIMIENTO Y CARACTERÍSTICAS MORFOLÓGICAS RELACIONADAS CON TIPO DE PLANTA ERECTA EN FRIJOL PARA RIEGO
}

\author{
SEED YIELD AND MORPHOLOGICAL TRAITS RELATED TO ERECT PLANT TYPE IN \\ IRRIGATED COMMON BEAN
}

\author{
Rafael A. Salinas Pérez ${ }^{1}$, Jorge A. Acosta Gallegos ${ }^{1 *}$, Ernesto López Salinas ${ }^{1}$, Ciria A. Torres Estrada ${ }^{1}$, \\ Francisco J. Ibarra Pérez ${ }^{1}$ y Rubén Félix Gastelum ${ }^{2}$
}

\begin{abstract}
${ }^{1}$ Programa de Frijol, Campo Experimental Bajío, Instituto Nacional de Investigaciones Forestales, Agrícolas y Pecuarias. Apdo. Postal 112. 34000 , Celaya, Gto. Tel 01 (461) 6115323 Ext. 200, Fax 01 (461) 6115431. ${ }^{2}$ Departamento de Ciencias Biológicas, Universidad de Occidente. Carretera Internacional y Boulevard Macario Gaxiola s/n. 81223, Los Mochis, Sinaloa.
\end{abstract}

*Autor para correspondencia (jamk@prodigy.net.mx)

\section{RESUMEN}

Para realizar la cosecha directa de frijol (Phaseolus vulgaris L.) se requieren plantas con crecimiento erecto. Durante el otoñoinvierno 2004 - 2005 se evaluaron 64 genotipos de cinco tipos de frijol: 'Azufrado', 'Pinto', 'Flor de Mayo', 'Flor de Junio' y 'Negro'. Los genotipos fueron de hábito determinado (tipo I) e indeterminado (tipos II y III) y se establecieron bajo riego en tres localidades: Los Mochis, Sinaloa; Celaya, Guanajuato y Cotaxtla, Veracruz, México. Se utilizó un diseño látice triple $8 \times 8$; la parcela fue un surco de $0.8 \mathrm{~m}$ de ancho y $6.0 \mathrm{~m}$ de longitud. Se determinaron siete características, dos relacionadas con la fenología, cuatro con la estructura de planta, y rendimiento. Para rendimiento hubo diferencias significativas $(P \leq \mathbf{0 . 0 5})$ entre sitios, genotipos $\mathrm{e}$ interacción. En Los Mochis el rendimiento varió de 1076 a $2534 \mathrm{~kg}$ $\mathrm{ha}^{-1}$, en Cotaxtla de 700 a $2276 \mathrm{~kg} \mathrm{ha}^{-1}$ y en Celaya de 800 a $4610 \mathrm{~kg}$ ha $^{-1}$. Esa variación fue consecuencia de la amplia diversidad en el origen y hábito de crecimiento de los genotipos, los que difirieron en grado de adaptación. En las tres localidades el rendimiento de los genotipos de hábito indeterminado tipos II y III superó al de las variedades determinadas tipo I. Las características altura de la primera vaina, madurez, acame de planta y grosor del hipocótilo se relacionaron entre sí: a mayor altura de la primera vaina, mayor grosor del hipocótilo y más días a madurez; mientras que a menor altura hubo, mayor porcentaje de acame. Un mayor acame se relacionó con menor grosor del hipocótilo. Ninguna de esas características mostró relación con el rendimiento. Bajo el sistema de siembra utilizado, los genotipos de planta erecta mostraron bajo rendimiento; entre éstos los sobresalientes fueron: A 525, 'Negro Nayarit', 'Negro Tacaná' y 'Negro Citlali', de hábito indeterminado Tipo II.

Palabras clave: Phaseolus vulgaris, altura de planta, grosor del hipocótilo, rendimiento.

\section{SUMMARY}

Direct harvest of common bean (Phaseolus vulgaris L.) is feasible, for that plants of erect growth habit are needed. A trial with 64 commercial bean genotypes was conducted during the FallWinter season 2004 - 2005. Tested genotypes including five commercial classes: 'Yellow', 'Pinto', 'Flor de Mayo', 'Flor de Junio' and 'Black', and three growth habits: determinate (type I) and indeterminate (types II and III). The trials were established under irrigated conditions at Los Mochis, in Sinaloa, Celaya, in Guanajuato and Cotaxtla, in Veracruz, México. A triple lattice design $8 \times 8$ was utilized and the experimental plot was a single 6-m row, with rows separated at $0.8 \mathrm{~m}$. During the growth cycle seven characteristics were recorded: two related to plant phenology, four to plant height structure, plus seed yield. For seed yield there were significant differences $(P>\mathbf{0 . 0 5})$ among sites and genotypes and for the genotype by site interaction. In Los Mochis seed yield varied from 1076 to $2534 \mathrm{~kg} \mathrm{ha}^{-1}$, in Cotaxtla from 700 to $2276 \mathrm{~kg} \mathrm{ha}^{-1}$ and in Celaya from 800 to $4610 \mathrm{~kg} \mathrm{ha}^{-1}$. This large variation observed in yield was the result of the wide diversity among genotypes regarding their origin and growth habit, which resulted in differences in adaptation. On all sites indeterminate genotypes of growth habit types II and III gave higher yields than determinate genotypes type I. The studied morphological traits resulted interrelated among themselves, height to the first pod, plant lodging, physiological maturity and hypocotyl thickness; the higher the first pod, the later to mature and thicker hypocotyls, whereas the lower the first pod, the higher plant lodging. None of these traits was related to seed yield. Under the utilized production system the genotypes of erect plant type displayed low yield; among them, those with high yield were: A 525, 'Negro Nayarit', 'Negro Tacaná' and 'Negro Citlali', all of indeterminate growth habit type II.

Index words: Phaseolus vulgaris, plant height, hypocotyl thickness, seed yield.

\section{INTRODUCCIÓN}

El cultivo del frijol (Phaseolus vulgaris L.) en el sistema de riego en México, es una excelente alternativa de producción; sin embargo, la escasez de la mano de obra para la cosecha y la necesidad de una pronta recolección son los principales problemas para de los productores. Una alternativa viable para solucionar esta problemática, es la generación de cultivares mejorados de planta erecta, que 
faciliten la cosecha mecánica directa, principalmente de las clases comerciales de alta demanda como: 'Peruano', 'Flor de Mayo', 'Flor de Junio', 'Pinto' y 'Negro' (Castellanos et al., 1997).

Algunas características de la planta de frijol se consideran componentes importantes en el desarrollo de cultivares con plantas erectas, como: grosor y altura del hipocótilo (Acquaah et al., 1991), ángulo de inserción de las ramas y número reducido de ramas por planta (Adams, 1982), altura de inserción de la primera vaina y longitud de entrenudos (Teixeira et al., 1999). Tales características se asocian principalmente con variedades de hábito indeterminado tipo II, de grano pequeño (Kelly y Adams, 1987; Brothers y Kelly, 1993).

Los caracteres morfológicos relacionados con arquitectura de planta erecta deben ser identificados en cada variedad porque son de herencia compleja, o bien que están ligados con un color o tamaño de grano determinado (Kelly y Adams, 1987; Acquaah et al., 1991). Por ello, la identificación de materiales sobresalientes en los tipos de frijol importantes en México facilitará el desarrollo de cultivares de arquitectura erecta. Los caracteres relacionados con planta erecta, podrían emplearse como criterios de selección en los programas de mejoramiento genético. La transferencia de la arquitectura erecta de materiales tropicales de hábito II y semilla pequeña a materiales de grano pinto con hábito indeterminado postrado tipo III, de semilla mediana, tomó tres ciclos de selección recurrente (Kelly y Adams, 1987), lo que sugiere asociaciones negativas entre las características de planta erecta y el tamaño de la semilla (Sexton et al., 1994). La evaluación de plantas individuales por arquitectura erecta no fue eficiente, y de entre los caracteres morfológicos estudiados, la longitud de los entrenudos mostró la mayor variación y predominancia de efectos aditivos (Teixeira et al., 1999).

Los objetivos de este estudio fueron: a) cuantificar el rendimiento de 64 genotipos de frijol de diferente hábito de crecimiento y origen en tres localidades, b) evaluar la relación de cinco características morfológicas con el tipo de planta erecta $\mathrm{y}, \mathrm{c})$ determinar la relación de esas características morfológicas con el rendimiento.

\section{MATERIALES Y MÉTODOS}

\section{Localidades de prueba}

Las evaluaciones experimentales se hicieron durante el ciclo de otoño-invierno 2004 - 2005 en condiciones de riego, en tres localidades: Los Mochis, Sinaloa en el trópico seco; Celaya, Guanajuato, en la región de El
Bajío; y en Cotaxtla, Veracruz, en el trópico húmedo. La siembra en Los Mochis fue el 25 de octubre de 2004, en Cotaxtla el 18 de octubre de 2004 y el 17 de marzo del 2005 en Celaya. Las características de las localidades de prueba se muestran en el Cuadro 1. Por lo general el clima de estas localidades en la estación de crecimiento es seco y libre de heladas, aunque con cierto riesgo de ocurrencia en Los Mochis por su localización al norte del país.

\section{Germoplasma utilizado}

Se estudiaron 64 genotipos de cinco tipos de frijol: 'Azufrado', 'Pinto', 'Flor de Mayo', 'Flor de Junio' y 'Negro' (Cuadro 2). Para cada clase y tipo de hábito de crecimiento se incluyó al menos una variedad testigo: 'Azufrado Higuera' (tipo I), 'Negro INIFAP' (tipo II), 'Flor de Mayo Anita' (tipo III) y 'Flor de Junio Silvia' (tipo III), respectivamente; así como dos líneas con arquitectura erecta generadas en el Programa de Frijol del Centro Internacional de Agricultura Tropical: A 55 (Singh et al., 2003) y A 525 (CIAT, 1995). La mayoría de los materiales evaluados provienen del proyecto de mejoramiento del Campo Experimental Valle del Fuerte, $\mathrm{y}$ algunos de otras sedes de mejoramiento genético del frijol del Instituto Nacional de Investigaciones Forestales, Agrícolas y Pecuarias (INIFAP) en México y presentan hábito de crecimiento determinado (tipo I) o indeterminado (tipos II y III) (Cuadro 2).

\section{Diseño experimental y conducción del ensayo}

Se utilizó un diseño experimental látice triple $8 \times 8$, con tres repeticiones, con parcela experimental de un surco de $0.8 \mathrm{~m}$ de ancho y $6.0 \mathrm{~m}$ de longitud; la parcela útil constó de $5 \mathrm{~m}$ centrales del surco $\left(4.0 \mathrm{~m}^{2}\right)$. Se fertilizó al momento de la siembra con la dosis 50N-50P$00 \mathrm{~K}$ de $\mathrm{N}_{2}-\mathrm{P}_{2} \mathrm{O}_{5}-\mathrm{K}_{2} \mathrm{O}_{5}$. En cada localidad se aplicaron los riegos necesarios y se dieron las labores agronómicas, de acuerdo con las recomendaciones para el cultivo de frijol de riego en la región (López et al., 2001; CEVAF, 2003).

\section{Características determinadas evaluadas}

Se consideraron seis características: dos relacionadas con la fenología de la planta, cuatro con la arquitectura de planta erecta, y rendimiento de grano. Las características fenológicas que se determinaron a partir de la siembra fueron: días a inicio floración y a madurez fisiológica. Las características morfológicas incluyeron: grosor del hipocótilo, acame de planta, hábito de crecimiento y altura de la primera vaina. La primera se midió con un vernier; la segunda en forma visual con una escala de 1 a 4 , donde 1 fue sin acame y 4 todas las plantas del surco 
acamadas; la tercera en forma visual conforme a la descripción de Singh (1982); y la altura de la primera vaina con una regla a partir de la superficie del suelo. El rendimiento de grano se determinó en un surco de $5 \mathrm{~m}$ de longitud, previa eliminación de $0.5 \mathrm{~m}$ de cabecera en cada parcela.

Cuadro 1. Características geográficas y climáticas de las localidades de prueba incluidas en el presente estudio.

\begin{tabular}{lccccccc}
\hline Localidad & $\begin{array}{c}\text { Temperatura } \\
\text { media }\left({ }^{\circ} \mathrm{C}\right)^{\dagger}\end{array}$ & $\begin{array}{c}\text { Altitud } \\
(\mathrm{m})\end{array}$ & $\begin{array}{c}\text { Precipitación } \\
\text { anual }(\mathrm{mm})\end{array}$ & $\begin{array}{c}\text { Latitud } \\
\text { Norte }\end{array}$ & $\begin{array}{c}\text { Longitud } \\
\text { Oeste }\end{array}$ & Tipo de suelo & Región climática \\
\hline $\begin{array}{l}\text { Los Mochis, } \\
\text { Sinaloa }\end{array}$ & 18.0 & 15 & 352 & $25^{\circ} 05^{\prime}$ & $108^{\circ} 38^{\prime}$ & Vertisol & Trópico seco \\
Celaya, Guanajuato & 20.6 & 1765 & 600 & $20^{\circ} 34^{\prime}$ & $100^{\circ} 50^{\prime}$ & Vertisol & Templado subhúmedo \\
Cotaxtla, Veracruz & 28.0 & 70 & 1400 & $18^{\circ} 50^{\prime}$ & $96^{\circ} 10^{\prime}$ & Franco arenoso & Trópico húmedo \\
\hline
\end{tabular}

${ }^{\dagger}$ Temperatura media durante el ciclo del cultivo.

Cuadro 2. Tipo de grano y hábito de crecimiento de los 64 genotipos de frijol aquí estudiados.

\begin{tabular}{|c|c|c|c|c|c|}
\hline Genotipo & Tipo $^{\dagger}$ & Hábito $^{\dagger \dagger}$ & Genotipo & Tipo & Hábito \\
\hline A 55 & $\mathrm{~N}$ & II & AP78/Mo-91-92-2029-4-M-M-M-3M & A & I \\
\hline 'Negro Sahuatoba' & $\mathrm{N}$ & III & AR87//(Azpa5/(II5FRM4-MMM//(G22242/XAN143) & A & I \\
\hline 'Negro Altiplano’ & $\mathrm{N}$ & III & AN/Mo-91-92-2029-2-2-2M & A & I \\
\hline 'Negro INIFAP' (T) & $\mathrm{N}$ & II & AP78/AP87/CCTM 19350-9-1-M-2M & A & III \\
\hline 'Negro Cotaxtla-91' & $\mathrm{N}$ & II & AZPA 5/Montcalm & A & I \\
\hline 'Negro Medellín’ & $\mathrm{N}$ & II & Mo-94-95-1740-9-CM-1-1-1M & A & III \\
\hline 'Negro Nayarit' & $\mathrm{N}$ & II & AZPA5/Can-100-6-1-2-2M2 & A & I \\
\hline 'Negro Tacaná' & $\mathrm{N}$ & II & Azufrado Higuera (T) & A & I \\
\hline A 525 & $\mathrm{C}$ & II & Azufrado Regional 87 & A & I \\
\hline AP78/Mo-91-92-2029-9M & A & III & Azufrado Peruano 87 & A & I \\
\hline AP78/Mo-91-92-2029-9M & A & II & AZPA 31 & A & II \\
\hline AZPA 231 & A & I & AFR-88/WAF-9 CCTM14820-M-1-CM & $\mathrm{C}$ & III \\
\hline AZPA 230 & A & I & PVA-721/G13094DR17675-5M & $\mathrm{C}$ & II \\
\hline AZPA 242 & A & I & (Mo-95-96-3413/AAM33)-6-1-1 & A & II \\
\hline AZPA 213 & A & I & PIMPA-11 & $\mathrm{P}$ & III \\
\hline AZPA 288 & A & I & Pinto Bayacora & $P$ & III \\
\hline AZPA 260 & A & II & 97-RS-101 & $\mathrm{P}$ & II \\
\hline AZPA 278 & A & II & 97-RS-110 & $P$ & II \\
\hline AZPA 280 & A & II & PR 96003-22 & $P$ & III \\
\hline AZPA 292 & A & II & Flor de Mayo P-17 & $\mathrm{FM}$ & II \\
\hline
\end{tabular}

${ }^{\dagger}$ Tipo de grano: $\mathrm{A}=$ Azufrado, $\mathrm{N}=$ Negro, $\mathrm{C}=$ Color claro $\mathrm{P}=$ Pinto, $\mathrm{FM}=$ Flor de Mayo, FJ $=$ Flor de Junio; ${ }^{\dagger \dagger}$ Hábito de crecimiento: $\mathrm{I}=$ Determinado, II = Indeterminado semi-guía, y III = Indeterminado guía postrado; $\mathrm{T}=$ variedad testigo. 


\section{Análisis estadísticos}

Las variables fueron sometidas a análisis de varianza por localidad y combinado a través de localidades, de correlación simple, y a la prueba de comparación de medias, con la prueba de Tukey $(\mathrm{P} \leq 0.05)$. Para los análisis se utilizó un programa estadístico para microcomputadoras MSTAT-C versión 2.1 (Freed et al., 1991). Para la discusión, los genotipos fueron agrupados por hábito de crecimiento.

\section{RESULTADOS Y DISCUSIÓN}

\section{Rendimiento y adaptación}

En el rendimiento hubo diferencias significativas $(\mathrm{P} \leq$ 0.05) entre localidades, genotipos e interacción genotipo por localidad (datos no mostrados). Los rendimientos en Celaya superaron a los de Los Mochis y de Cotaxtla (Cuadro 3). A pesar de que el ensayo se condujo en las tres localidades de prueba bajo las mismas condiciones de manejo, en riego y fertilización, la significancia de la interacción genotipo por ambiente confirma que diferentes genotipos son adecuados para cada localidad, como ocurre con las variedades comerciales que se recomiendan y explotan en cada región (Rosales et al., 2004). Por lo anterior, los mejores genotipos en cada localidad fueron diferentes. Sabaghnia et al. (2006) han señalado en otras leguminosas de grano, las dificultades inherentes en la selección por efecto de la interacción genotipo por ambiente.

En general, los genotipos de hábito de crecimiento indeterminado (tipos II y III) resultaron superiores a los de hábito determinado (tipo I) en las tres localidades, diferencia que fue acentuada en Celaya (Cuadro 3). El menor potencial de rendimiento de los genotipos de hábito determinado, entre otros factores, se debe a su precocidad (Cuadro 4) y a la relación negativa entre tamaño de la semilla y potencial de rendimiento (Park, 1993; Sexton et al., 1994). En Los Mochis el rendimiento promedio por hábito de crecimiento resultó relativamente similar, lo que en parte explica la preferencia por variedades de hábito determinado tipo I en esa región, ya que plantas de este tipo facilitan las labores mecanizadas y el riego. Por otra parte, en la región tropical húmeda, como en Cotaxtla, se prefieren las variedades de hábito tipo II porque son de porte erecto en comparación con los de tipo III, por que en estas últimas pueden ocurrir pérdidas considerables por manchado y pudrición del grano al estar las vainas en contacto con el suelo. En Cotaxtla el rendimiento de los tipos II y III resultó fue similar (Cuadro 3). En Celaya, la principal variedad en condiciones de riego es 'Flor de Junio Marcela', de hábito indeterminado tipo III; en esta localidad, además de las variedades de tipo III, las de tipo II mostraron excelente adaptación y rendimiento.

En Los Mochis los genotipos de mayor rendimiento fueron: PVA721/G13094-DR17675-5-M, Mo82-834044/ZAA-78-2-2, FMP 17, 'Flor de Mayo Anita', 97RS-110 y 97-RS-101 con rendimientos de 2 534, 2 429, $2325,2325,2257$ y $2187 \mathrm{~kg} \mathrm{ha}^{-1}$, respectivamente. Los dos primeros fueron desarrollados por el programa local y los cuatro restantes provienen del programa de Celaya y de ellos sólo el primero es de hábito tipo I y el resto es de tipo III (Cuadros 4, 5 y 6). En Cotaxtla los genotipos sobresalientes fueron: 'Negro Nayarit', DON 38, AP78/45DRM-4-M-M-M-3M， AP78/Mo-91-92-2029, 'Negro Cotaxtla 91' y 'Negro Citlali', con rendimientos de 2 301, 2 276, 2 184, 2 142, 2085 y $2048 \mathrm{~kg} \mathrm{ha}^{-1}$, respectivamente. Tres son de hábito indeterminado tipo II y dos de tipo III (Cuadros 5 y 6). De todos ellos, sólo 'Negro Cotaxtla' fue desarrollado en el programa local, tres son del programa de Los Mochis y dos del de Celaya. En Celaya los genotipos con mayor rendimiento fueron: Mo-82-83-4044/ZAA78-2-2, 97-RS-101, 'Negro Citlali', AP78/45DRM-4-M-M-M-3M y ANxMo91-92-2029-2-2$2 \mathrm{M}$; de éstos, dos son del programa local y tres del de Los Mochis; tres son de hábito indeterminado tipo III y dos de tipo II (Cuadros 5 y 6).

Los resultados anteriores indican que el intercambio de germoplasma entre programas de mejoramiento ubicados en diferentes regiones del país, es una posibilidad viable para ampliar la base genética de cada uno de ellos (Singh, 2001) y para la obtención de nuevas variedades por el método de introducción (Fehr, 1987); esto último en caso de que el material introducido cumpla con las características agronómicas relacionadas con la adaptación a la región y con las demandadas de los consumidores. En Celaya, con excepción de AZPA 230 (tipo I), los genotipos superiores sobresalientes fueron de hábito indeterminado (tipo II o III), lo que coincide con lo descrito por diversos investigadores (White et al., 1992; Park, 1993), referente a que el mayor potencial de rendimiento está asociado con el hábito de crecimiento indeterminado y en forma negativa con el tamaño de la semilla (White y Gonzalez, 1990). Así, el rendimiento promedio de las variedades correspondientes a los tipos II y III fue superior a las de tipo I, en las tres localidades de prueba (Cuadro 3).

Las líneas con tipo de planta erecta introducidas del CIAT, (A 55 y A 525) estuvieron entre las de mayor altura de planta y menor peso de 100 semillas, y su nivel de rendimiento fue intermedio; A 525 superó en rendimiento a A 55. Las variedades 'Negro Nayarit', 'Negro Tacaná' y 'Negro Citlali' de planta erecta tipo II 
estuvieron entre los genotipos de mayor rendimiento en las tres localidades de prueba, cuyo grano es de valor comercial. Todos los genotipos erectos son de semilla pequeña y negra, excepto A 525 que es de semilla de color claro; lo anterior significa que en México ya existen genotipos de planta erecta, pero sólo para el tipo comercial de grano negro, pequeño y opaco, como las variedades 'Negro Nayarit', 'Negro Tacaná' y 'Negro Citlali'.

El rendimiento varió de 1076 a $2534 \mathrm{~kg} \mathrm{ha}^{-1}$ en Los Mochis, de 700 a 2301 en Cotlaxtla, mientras que en Celaya osciló de 750 a $4550 \mathrm{~kg} \mathrm{ha}^{-1}$ (Cuadros 4, 5 y 6).
Estas amplias variaciones en rendimiento son congruentes con la amplia diversidad de genotipos incluidos en este estudio en cuanto a origen, hábito de crecimiento, ciclo de cultivo y resistencia a enfermedades, por lo cual difieren en su grado de adaptación a cada localidad especifica. La adaptación en parte fue conferida por el ciclo biológico y el nivel de resistencia a las enfermedades en cada localidad de prueba, sobre todo a las enfermedades que causan las pudriciones de raíz (Fusarium spp. y Rhizoctonia solani) y a tizón común (Xanthomonas campestris pv phaseoli), patógenos que estuvieron presentes en las tres localidades.

Cuadro 3. Rendimiento promedio y rango de rendimiento de 64 genotipos de frijol agrupados por hábito de crecimiento producidos en condiciones de riego en tres localidades.

\begin{tabular}{|c|c|c|c|c|c|}
\hline \multirow[t]{2}{*}{ Localidad } & \multirow[t]{2}{*}{ Rango } & \multirow{2}{*}{$\begin{array}{l}\text { Rendimiento } \\
\text { promedio }\left(\mathrm{kg} \mathrm{ha}^{-1}\right)\end{array}$} & \multicolumn{3}{|c|}{ Hábito de crecimiento } \\
\hline & & & Tipo I (16) $)^{\dagger \dagger}$ & Tipo II (24) & Tipo III (24) \\
\hline Los Mochis & $1076-2534$ & $1742 \mathrm{~b}^{\dagger}$ & 1666 & 1809 & 1726 \\
\hline Celaya & $750-4610$ & $2678 \mathrm{a}$ & 1997 & 2951 & 2942 \\
\hline Cotaxtla & $599-2301$ & $1331 \mathrm{~b}$ & 1187 & 1467 & 1480 \\
\hline Promedio & & 1917 & $1563 \mathrm{~B}$ & $1999 \mathrm{~A}$ & $2106 \mathrm{~A}$ \\
\hline
\end{tabular}

${ }^{\dagger}$ Medias con la misma letra dentro de columnas (minúsculas) o hileras (mayúsculas) son estadísticamente iguales (Tukey, 0.05)

†† Número de genotipos por tipo de hábito de crecimiento.

Cuadro 4. Características agronómicas de 16 genotipos de frijol de hábito determinado (tipo I) establecidos en tres localidades en condiciones de riego.

\begin{tabular}{|c|c|c|c|c|c|c|c|c|c|}
\hline \multirow[b]{2}{*}{ Genotipo } & \multicolumn{3}{|c|}{ Días a flor ${ }^{\dagger}$} & \multicolumn{3}{|c|}{ Días a madurez $^{\dagger}$} & \multicolumn{3}{|c|}{ Rendimiento ( $\left.\mathrm{kg} \mathrm{ha}^{-1}\right)$} \\
\hline & $\mathrm{Mo}^{\dagger \dagger}$ & $\mathrm{Ce}$ & Co & Mo & $\mathrm{Ce}$ & Co & Mo & $\mathrm{Ce}$ & Co \\
\hline AZPA 231 & 54 & 52 & 38 & 118 & 103 & 64 & 1492 & 3175 & 1364 \\
\hline AZPA 242 & 54 & 45 & 36 & 120 & 100 & 78 & 1389 & 1125 & 1244 \\
\hline AZPA-213 & 53 & 46 & 36 & 117 & 100 & 75 & 1389 & 2050 & 1759 \\
\hline AR87/VA566 (95-96) & 54 & 44 & 36 & 125 & 100 & 72 & 1215 & 1250 & 730 \\
\hline AZPA5/C100-6-1-2-2-M2 & 54 & 46 & 35 & 118 & 100 & 79 & 1770 & 2675 & 781 \\
\hline ‘Azufrado Higuera’ (T) & 44 & 45 & 36 & 120 & 100 & 73 & 1562 & 1625 & 1108 \\
\hline 'Azufrado Regional-87' & 39 & 51 & 35 & 120 & 100 & 79 & 1146 & 1500 & 1418 \\
\hline 'Azufrado Peruano-87' & 48 & 53 & - & 118 & 103 & - & 1423 & 1875 & $-\theta^{\dagger+\dagger}$ \\
\hline PVA721/G13094DR17675-5-M & 52 & 49 & 37 & 127 & 98 & 76 & 2534 & 1125 & 1370 \\
\hline 'Flor de Mayo P-2' & 63 & 45 & 41 & 124 & 103 & 83 & 1736 & 1275 & 1542 \\
\hline 'Rayado Rojo' & 54 & 52 & 35 & 125 & 100 & 66 & 1597 & 1475 & 1581 \\
\hline DMS (0.05) & 8.9 & 7.3 & 8.6 & 11 & 12.4 & 9.4 & 443 & 543 & 672 \\
\hline $\mathrm{CV}(\%)$ & 7.6 & 9.3 & 8.8 & 5.6 & 6.4 & 7.3 & 22.1 & 18.9 & 17.6 \\
\hline Promedio & & & & & & & 1666 & 1997 & 1187 \\
\hline
\end{tabular}

${ }^{\dagger}$ Días después de la siembra.

${ }^{\dagger}$ Localidades: $\mathrm{Mo}=$ Los Mochis, Sinaloa, Ce = Celaya, Guanajuato., Co = Cotaxtla, Veracruz.

${ }^{\dagger \dagger}$ No incluido en el ensayo. 
Con respecto a los testigos, se observó que 'Azufrado Higuera' de hábito determinado fue superado en rendimiento de grano por genotipos del mismo hábito en las tres localidades (Cuadro 4); 'Negro INIFAP' de hábito indeterminado tipo II, fue superado por la variedad 'Negro Nayarit' en dos localidades y por 'Negro Citlali' en Celaya, Gto. (Cuadro 5). Los testigos 'Flor de Junio Silvia' y 'Flor de Mayo Anita', ambos de hábito indeterminado tipo III, fueron superados por pocas líneas experimentales en las tres localidades (Cuadro 6).

En promedio, los 64 genotipos fueron más tardíos en Los Mochis, y más precoces en Cotaxtla donde se registró el menor numero de días a madurez fisiológica (Cuadros 4, 5 y 6); esa respuesta a través de localidades fue debida a las diferentes temperaturas ocurridas en cada localidad durante el ciclo del cultivo, que fueron más altas en Cotaxtla y más bajas en Los Mochis. El ciclo de cultivo se redujo en Cotaxtla, lo cual explica el menor rendimiento promedio en esa localidad; el mayor ciclo de cultivo observado en Los Mochis sugiere la ocurrencia de temperaturas bajas (subóptimas) durante el ciclo, temperaturas que no condujeron a obtener altos rendimientos pero si a un ciclo de cultivo más largo (Wallace et al., 1993).

Cuadro 5. Características agronómicas de 24 genotipos de frijol de hábito indeterminado (tipo II) establecidos en tres localidades en condiciones de riego.

\begin{tabular}{|c|c|c|c|c|c|c|c|c|c|}
\hline \multirow{2}{*}{ Genotipo } & \multicolumn{3}{|c|}{ Días a flor ${ }^{\dagger}$} & \multicolumn{3}{|c|}{ Días a madurez $^{\dagger}$} & \multicolumn{3}{|c|}{ Rendimiento $\left(\mathrm{kg} \mathrm{ha}^{-1}\right)$} \\
\hline & $\mathrm{Mo}^{\dagger \dagger}$ & $\mathrm{Ce}$ & Co & Mo & $\mathrm{Ce}$ & $\mathrm{Co}$ & Mo & $\mathrm{Ce}$ & Co \\
\hline A 55 & 58 & 55 & 41 & 122 & 100 & 77 & 1631 & 2425 & 949 \\
\hline 'Negro Cotaxtla-91' & 63 & 58 & 40 & 124 & 105 & 76 & 1076 & 3425 & 2085 \\
\hline ‘Negro Medellín’ & 62 & 56 & 39 & 120 & 103 & 75 & 1354 & 3550 & 1778 \\
\hline 'Negro Nayarit' & 62 & 54 & 41 & 122 & 103 & 82 & 2153 & 3775 & 2301 \\
\hline A 525 & 62 & 55 & 43 & 129 & 103 & 85 & 1631 & 2375 & 1528 \\
\hline AP78/Mo-91-92-2029-9M & 59 & 55 & 39 & 125 & 103 & 75 & 1874 & 3725 & 1351 \\
\hline AP78/Mo-91-92-2029-19M & 55 & 45 & 37 & 123 & 103 & 72 & 1527 & 3025 & 1019 \\
\hline C72//(C72xAAm33/C72) & 55 & 49 & 36 & 115 & 98 & 78 & 2082 & 3300 & 1284 \\
\hline AZPA 288 & 56 & 54 & 40 & 117 & 100 & 84 & 1423 & 2750 & 1624 \\
\hline AZPA 292 & 54 & 53 & 42 & 123 & 103 & 82 & 2186 & 3000 & 1176 \\
\hline AP78/Mo-91-92-1585 & 56 & 55 & 41 & 126 & 105 & 80 & 2152 & 3000 & 1160 \\
\hline AP78/Mo-91-92-2029-4-M-M-M-3M & 54 & 42 & 38 & 125 & 98 & 79 & 2152 & 800 & 971 \\
\hline $\begin{array}{l}\text { AR87/(AZPA5/(II5FrM4-MMM } \\
\text { //(G22242/XAN143) }\end{array}$ & 56 & 57 & 40 & 123 & 105 & 81 & 1840 & 750 & 1745 \\
\hline ANxMo-91-92-2029-2-2-2M & 56 & 45 & 44 & 117 & 100 & 82 & 1666 & 4125 & 941 \\
\hline AZPA-31 & 49 & 52 & - & 117 & 100 & - & 1979 & 3700 & - \\
\hline PVA-721/G13094DR17675-5M & 54 & 47 & 42 & 119 & 100 & 78 & 1527 & 1000 & 1294 \\
\hline 97-RS-101 & 53 & 52 & - & 118 & 103 & - & 2187 & 4500 & - \\
\hline 97-RS-110 & 54 & 50 & 36 & 118 & 103 & 71 & 2257 & 1125 & 1126 \\
\hline
\end{tabular}

\footnotetext{
${ }^{\dagger}$ Días después de la siembra.
}

\#Localidades: Mo = Los Mochis, Sinaloa, $\mathrm{Ce}=$ Celaya, Guanajuato, Co $=$ Cotaxtla, Veracruz. 
Cuadro 6. Características agronómicas de 24 genotipos de frijol de hábito indeterminado tipo III establecidos en tres localidades en condiciones de riego.

\begin{tabular}{|c|c|c|c|c|c|c|c|c|c|}
\hline \multirow{2}{*}{ Genotipo } & \multicolumn{3}{|c|}{ Días a flor ${ }^{\dagger}$} & \multicolumn{3}{|c|}{ Días a madurez ${ }^{\dagger}$} & \multicolumn{3}{|c|}{ Rendimiento $\left(\mathrm{kg} \mathrm{ha}^{-1}\right)$} \\
\hline & $\mathrm{Mo}^{\dagger \dagger}$ & $\mathrm{Ce}$ & Co & Mo & $\mathrm{Ce}$ & $\mathrm{Co}$ & Mo & $\mathrm{Ce}$ & Co \\
\hline 'Negro Sahuatoba' & 63 & 59 & 41 & 123 & 105 & 81 & 1457 & 3325 & 1769 \\
\hline 'Negro Altiplano' & 57 & 54 & 36 & 122 & 103 & 78 & 2082 & 2750 & 1528 \\
\hline 'Jamapa Plus' & - & 57 & 42 & - & 103 & 77 & - & 3125 & 1129 \\
\hline AP78/Mo-91-92-2029-9M & 54 & 48 & 37 & 125 & 97 & 80 & 1770 & 2800 & 1195 \\
\hline II5FRM-4-M-M-M/AAm33 & 60 & 54 & 43 & 124 & 103 & 85 & 1666 & 3175 & 855 \\
\hline Mo-82-83-4044/ZAA 78-2-2 & 59 & 52 & 42 & 117 & 103 & 82 & 2429 & 4550 & 911 \\
\hline AR87/1963 (95-96) & 56 & 54 & 38 & 115 & 105 & 80 & 1874 & 2675 & 1413 \\
\hline AP78/45DRM-4-M-M-M-3M & 56 & 54 & 39 & 117 & 105 & 82 & 1666 & 4400 & 2184 \\
\hline AP78/C72-36-M-M-M & 56 & 55 & 41 & 125 & 105 & 81 & 2071 & 3000 & 1831 \\
\hline PP80/CL-3-M-M-M & 56 & 49 & 35 & 127 & 100 & 80 & 1735 & 3900 & 1825 \\
\hline Comp-Mo-1/Mo-91-92-2029 & 55 & 46 & 38 & 126 & 100 & 72 & 1840 & 3800 & 919 \\
\hline AP78/AP-87/CCTM 19350-9-1-M-2M & 61 & 53 & 40 & 116 & 103 & 85 & 1284 & 2750 & 723 \\
\hline Mo-94-95-1740-9-CM-1-1-1-M & 56 & 52 & 37 & 128 & 103 & 72 & 1840 & 2625 & 1251 \\
\hline AP78/Mo-91-92-2029-20-M & 55 & 55 & 40 & 124 & 105 & 80 & 1666 & 2125 & 2142 \\
\hline PVA721/G13094DR17675-6-M & 54 & 53 & 42 & 127 & 103 & 79 & 1551 & 2300 & 1247 \\
\hline AR87/CL-20-2-CM-M & 54 & 52 & 38 & 124 & 100 & 73 & 1632 & 775 & 1346 \\
\hline AFR-88/WAF-9 CCTM14820-M-1-M & 55 & 53 & 37 & 118 & 105 & 70 & 2013 & 2025 & 1193 \\
\hline PIMPA-11 & 54 & 45 & 36 & 118 & 100 & 66 & 1528 & 3575 & 1085 \\
\hline 'Pinto Bayacora' & 52 & 43 & - & 117 & 95 & - & 1597 & 1500 & - \\
\hline PR 96003-22 & 55 & 54 & 40 & 124 & 103 & 64 & 1076 & 1350 & 1654 \\
\hline 'Flor de Junio Silvia' & 61 & 55 & 37 & 129 & 105 & 81 & 1458 & 3425 & 1636 \\
\hline 'Flor de Mayo Anita' & 62 & 58 & 40 & 120 & 100 & 84 & 2325 & 3875 & 1525 \\
\hline DON 38 & 62 & 52 & 39 & 124 & 103 & 79 & 1285 & 3850 & 2276 \\
\hline C V (\%) & 5.6 & 6.4 & 5.4 & 7.8 & 6.9 & 6.7 & 17.5 & 20.3 & 21.4 \\
\hline DMS (0.05) & 6.8 & 6.2 & 7.0 & 9.1 & 8.2 & 6.5 & 634 & 742 & 782 \\
\hline Promedio & & & & & & & 1726 & 2942 & 1480 \\
\hline
\end{tabular}

${ }^{\dagger}$ Número de días después de la siembra.

${ }^{\dagger}$ Localidades: $\mathrm{Mo}=$ Los Mochis, Sinaloa, $\mathrm{Ce}=$ Celaya, Guanajuato, $\mathrm{Co}=$ Cotaxtla, Veracruz.

Cuadro 7. Correlaciones simples entre el rendimiento y características agronómicas relacionadas con la arquitectura erecta en frijol bajo riego en dos localidades, Los Mochis, Sinaloa y Cotaxtla, Veracruz.

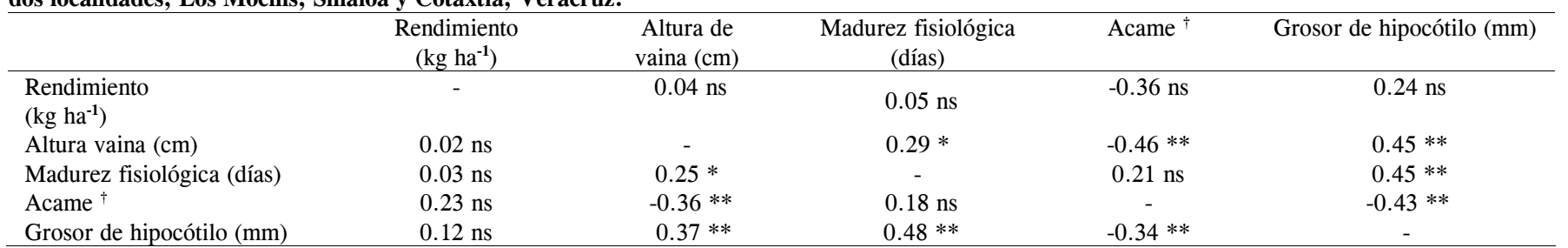

*, ** Significativo, 0.05 y 0.01 , respectivamente y $\mathrm{ns}=$ no significativo. Los valores arriba de la diagonal corresponden a la localidad de Los Mochis, Sinaloa, y los de abajo a Cotaxtla, Veracruz.

${ }^{\dagger}$ Se utilizó una escala visual de 1 a $4,1=$ sin acame, 4 = todas las plantas acamadas. 


\section{Características morfológicas}

Para poder desarrollar cultivares de frijol con planta erecta, primero es necesario identificar las características morfológicas asociadas, mismas que pueden diferir entre tipos de frijol, sobre todo entre variedades con diferente tamaño de grano (Teixeira et al., 1999). Adams (1973) estableció que las variedades con arquitectura ideal deberán poseer un tallo fuerte y grueso, de tal forma que el transporte interno de nutrientes sea eficiente y que las plantas sean resistentes al acame. Con respecto a las características morfológicaos evaluadas en Los Mochis y Cotaxtla, se detectaron diferencias significativas ( $\mathrm{P} \leq$ 0.05 ) en las seis características consideradas; dos fenológicas: días a flor y a madurez fisiológica, tres de tipo morfológico: grosor del hipocótilo, altura de la primera vaina y acame. Estas características pueden considerarse importantes en relación a la arquitectura de planta erecta, sobre todo el grosor del hipocótilo, característica que ha mostrado heredabilidad intermedia y efectos aditivos en su control (Acosta et al., 1988; Acquua et al., 1991), mientras que la altura de la primera vaina es importante para la cosecha directa. También la longitud del entrenudo se considera una característica importante en el desarrollo de cultivares de planta erecta (Teixeira et al., (1999).

\section{Relación entre características}

Las relaciones entre caracteres morfológicos fueron las siguientes: a mayor número de días a madurez, mayor altura de la vaina y grosor del hipocótilo; la altura de la vaina mostró una relación inversa con el acame $(\mathrm{P} \leq$ 0.01 ), es decir a menor altura de la vaina, mayor porcentaje de acame de la planta (Cuadro 7). El acame presentó una relación inversa con el grosor del hipocótilo $(\mathrm{P} \leq 0.01)$, a mayor acame menor grosor del hipocótilo. Como fue señalado por otros autores (Adams, 1982; Acquua et al., 1991), a través de estos caracteres morfológicos se establecen los criterios para la identificación de prototipos de planta de arquitectura erecta; sin embargo, en los sistemas de producción utilizados, ninguno de ellos se relacionó con el rendimiento. Para lograr un mayor rendimiento con genotipos erectos, sobre todo de los de hábito determinado, se requiere de un balance adecuado de los caracteres asociados al tipo de planta erecta y los relacionados con alto rendimiento; otra posibilidad es la modificación del sistema de siembra para acomodar un mayor numero de plantas por unidad de área (Park, 1993). En un estudio de heredabilidad por Teixeira et al. (1999), de un grupo de caracteres relacionados con la arquitectura de planta erecta en frijol, la longitud de los entrenudos mostró amplia variación y los efectos aditivos fueron preponderantes en el control del carácter. Efectos similares han sido señalados para el grosor del tallo en frijol de temporal o secano (Acosta et al., 1988).

El acame fue la única característica morfológica que mostró ligera asociación con el rendimiento (Cuadro 7), lo que parece explicable si se considera que las variedades de hábito indeterminado postrado tipo III, por lo general fueron de alto rendimiento y con ramas y vainas que tocan el suelo, lo cual pudiera considerarse como problema de acame. Se considera que plantas del tipo III no son adecuadas para la cosecha directa, pero su cosecha mecanizada se realiza mediante varios pasos de maquinaria. Las variedades de hábito indeterminado arbustivo tipo II, se consideran con un tipo de planta erecta fisiológicamente eficiente (Adams, 1973; Adams, 1982; Kelly y Adams, 1987; Acquaah et al., 1991), pero que generalmente poseen semillas pequeñas (Teixeira $e t$ al.,1999) y se ha observado un alto grado de dificultad en el desarrollo de variedades de habito indeterminado y erectas con semillas de tamaño intermedio y grande (Kelly y Adams, 1987; Brothers y Kelly, 1993).

Los resultados sugieren que los materiales sobresalientes en tipo de planta erecta y rendimiento aceptable, como A 525, 'Negro Nayarit', 'Negro Tacana' y 'Negro Citlali', deben utilizarse en cruzamientos con los materiales sobresalientes en rendimiento en los tipos comerciales de interés. Es probable que en las diferentes localidades, distintos progenitores sean los adecuados. Los tres últimos genotipos poseen grano de valor comercial y pueden utilizarse en las regiones donde el grano de color negro opaco es aceptado por los consumidores.

En el proceso de selección, las características como grosor del hipocótilo o del tallo y longitud de los entrenudos pueden ser utilizados como criterios de selección en generaciones intermedias, ya que su efectividad con base en plantas individuales en generaciones tempranas presenta bajos niveles de heredabilidad (Teixiera et al., 1999). Entre los materiales de hábito determinado, que aun con menor altura de planta que los arriba mencionados, también pueden utilizarse como progenitores e inclusive ser cosechados en forma directa, se identifico material de con rendimiento aceptable, se identificaron las líneas: AZPA5/Can-100-61-2-2-M2, (Mo-95-96-3413/AAm33)-6-1-1, AZPA5/ Montcalm y AZPA 230, que superaron a los testigos a través de las localidades de prueba y cuyo grano es de valor comercial en Sinaloa y Guanajuato.

Se considera que la distancia entre surcos utilizada en las pruebas realizadas, no fue la adecuada para la 
obtención de alto rendimiento con plantas erectas o de hábito determinado, cuya capacidad competitiva es reducida. Es decir, los materiales sobresalientes en tipo de planta erecta deberán ser evaluados en altas densidades (Park, 1993; Teixeira et al., 1999), ya sea en surcos más estrechos que los aquí utilizados o bien en camas con doble o triple hilera para determinar su potencial de rendimiento bajo esos sistemas de producción.

\section{CONCLUSIONES}

A través de localidades, diferentes genotipos de frijol de hábito de crecimiento indeterminado fueron los de mayor rendimiento, en casi todos los casos. En cada localidad se identificaron genotipos introducidos con alto potencial de rendimiento.

Los caracteres morfológicos relacionados con la arquitectura de planta erecta: altura de la primera vaina, días a madurez fisiológica, acame de planta y grosor del hipocótilo, no mostraron relación con el rendimiento. Por el contrario, el acame de la planta mostró relación negativa significativa con el rendimiento.

Los genotipos de planta erecta con mayor rendimiento a través de localidades fueron de hábito indeterminado tipo II, A 525, 'Negro Nayarit', 'Negro Tacaná' y 'Negro Citali’, todos de grano pequeño.

\section{BIBLIOGRAFÍA}

Acosta G J A, R Ochoa M, I Sánchez V (1988) Efecto del genotipo y del ambiente en algunas características agronómicas del frijol de temporal. Agric. Téc. Méx. 14:1-15.

Acquaah G, M W Adams, J D Kelly (1991) Identification of effective indication of erect plant architecture in dry bean ideotype. Crop Sci. 31:261-264.

Adams M W (1973) Plant architecture and physiological efficiency in the field bean. In: Potentials of Field Beans and other Food Legumes in Latin America. Series Seminars No. 2E, Cali, Colombia (Centro Internacional de Agricultura Tropical) pp:266-278.

Adams M W (1982) Plant arquitecture and yield breeding in Phaseolus vulgaris L. Iowa State J. Res. 56:225-254.

Brothers M E, J D Kelly (1993) Interrelationship of plant architecture and yield components in the pinto bean ideotype. Crop Sci. $33: 1234-1238$.
Campo Experimental Valle del Fuerte, CEVAF (2003) Guía para la Asistencia Técnica Agrícola para el Área de Influencia del Campo Experimental Valle del Fuerte. INIFAP-CIRNO, Campo Experimental Valle del Fuerte. Agenda Técnica, 6a ed, Juan José Ríos, Sin. México. 208 p.

Castellanos J Z, H Guzmán-Maldonado, A Jiménez, C Mejía, J J Muñoz-Ramos, J A Acosta-Gallegos, G Hoyos, E LópezSalinas, D González, R Salinas-Pérez, J González-Acuña, J A Muñoz-Villalobos, P Fernández, B Cázares (1997) Hábitos preferenciales de los consumidores de frijol común (Phaseolus vulgaris L.) en México. Arch. Latinoam. Nutr. 47:163-168

Centro Internacional de Agricultura Tropical (1995) Catálogo de Líneas Avanzadas de Frijol del CIAT. A Rodríguez M, F Ramírez H, O Voysest y White J W (comps). CIAT, Cali, Colombia p.285 p.

Fehr W R (1987) Principles of Cultivar Development. Vol. I Theory and Technique. Macmillan Publishing Company, New York, U.S.A. pp:388-400

Freed R, S P Eisensmith, S Goetz, D Reicosky, U W Smail, P Wolberg (1991) User's Guide to MSATC. Michigan State University, East Lansing, Michigan.

Kelly J D, M W Adams (1987) Phenotypic recurrent selection in ideotype breeding of pinto beans. Euphytica 36:69-80.

Park S J (1993) Response of bush and upright plant type selections to white mold and seed yield of common bean in various row widths in Southern Ontario. Can. J. Plant Sci. 73:265-272

Sabaghnia N, H Deghhani, S S Hossain (2006) Nonparametric methods for interpreting genotype $\mathrm{x}$ environment interaction of lentil genotypes. Crop Sci. 46:1100-1106.

Sexton P J, J W White, K J Bote (1994) Yield-determining processes in relation to cultivar seed size of common bean. Crop Sci. 34:84-91.

Singh S P (1982) A key for identification of different growth habits of Phaseolus vulgaris L. Ann. Rep. Bean Improv. Coop. 25:9295

Singh S P (2001) Broadening the genetic base of common bean cultivars: A review. Crop. Sci. 41:1659-1675.

Singh S P, A Gutierrez, H Teran (2003) Registration of indeterminate tall upright small black seeded common bean germplasm A 55. Crop Sci. 43:1887-1888

Teixeira F F, A P Rarmalho, F A B Abreu (1999) Genetic control of plant architecture in the common bean. Genet. Mol. Biol. 22:577-582

Wallace D H, K S Yourstone, P N Masaya, R W Zobel (1993) Photoperiod gene control over partitioning between reproductive and vegetative growth. Theor. Appl. Genet. 86:6-16.

White J W, A Gonzalez (1990) Characterization of the negative association between seed yield and seed size among genotypes of common bean. Field Crops Res. 23:159-175

White J W, J Kornegay, J Castillo, C H Molano, C Cajiao, G Tejeda (1992) Effect of growth habit on yield of large-seeded bush cultivars of common bean. Field Crops Res. 29:151161 . 